\title{
Effect of hydrocolloid addition on batter properties and quality of deep-fried banana (Musa spp.) fritters
}

\begin{abstract}
Purpose: Deep-fried banana (Musa spp.) fritters is one of the frequently consumed fruit based snacks in Southeast Asian countries despite its substantial amount of oil content. Consistent with the demand for low fat food with maintained palatability, this study aimed to determine the quality of banana fritters as affected by batter system containing selected hydrocolloids such as pectin (PCN), whey protein isolate (WPI) and soy protein isolate (SPI). Design/methodology/approach: Banana fritter batters were prepared with individual addition of $2 \% \mathrm{PCN}(\mathrm{w} / \mathrm{w}), 10 \% \mathrm{WPI}(\mathrm{w} / \mathrm{w})$, $10 \%$ SPI (w/w), combination of $2 \%$ PCN and $10 \%$ WPI, combination of $2 \%$ PCN and $10 \%$ SPI and control (without hydrocolloid addition). Batter viscosity (Pa.s) and batter pick-up (\%) were determined. Banana fritters were analysed for moisture and fat contents, moisture loss, colour, hardness and sensory characteristics. Findings: Hydrocolloid addition in the batter system resulted in a higher batter pick-up and viscosity in comparison to control batter system. Moisture loss from banana fritters with batter formulation of $2 \%$ PCN and $10 \%$ SPI was the lowest while the reduction in oil content $(55 \%)$ was the highest. Banana fritters with inclusion of hydrocolloids in the batter formulation were equally accepted as the control sample by the sensory panelists with a score range between 6 and 7 for most of the sensory attributes evaluated except for oiliness. Originality/value: Application of PCN and SPI in batter system to develop banana fritters with low oil content, moist fruit core and crunchy crust is reported for the first time. Batter premix containing PCN and SPI can be produced for fresh and frozen fritters preparation.
\end{abstract}

Keyword: Oil absorption; Quality; Banana fritters; Pectin; Whey protein isolate; Soy protein isolate 